\title{
SCENSION AND DECLINE OF SUCCESSFUL COMPANIES:
A BIBLIOMETRIC STUDY ON THE ICARUS PARADOX
}

\author{
${ }^{1}$ Marcio Augusto Lassance Cunha Filho \\ ${ }^{2}$ Eliane Herrero \\ ${ }^{3}$ Claudia Cristina Martins de Mello \\ ${ }^{4}$ Douglas Vidal
}

\begin{abstract}
Objective: Our main objective was to examine the extension of published articles that cite Danny Miller's (1990) book, The Icarus Paradox, and to identify the current research fronts that use the book as a reference.

Methodology/approach: This is a bibliometric study with the technique of bibliographic coupling.

Originality / Relevance: The identification and understanding of the phenomena that can potentially lead some successful companies to failure are relevant issues for research on the social sciences areas, especially in the field of organizational strategy.
\end{abstract}

Main results: We identified five factors that encompass the main articles that refer to Miller's (1990) book. The themes transformational leadership and environment, competitive advantages, resources, processes of change and perception of opportunities represent the main currents of research that consider and refer to the findings of Danny Miller. These factors regard the research of organizational strategies for the preservation of competitive advantage, with identification of problems that can lead companies to failure.

Theoretical/methodological contributions: In our study, there is a practical and detailed guideline for bibliometric studies with analysis of citations and coupling, using the Exploratory Factor Analysis. Still, we hope that the description of the factors may guide future researchers through the identification of the research trends on the themes addressed.

Social/management contributions: Identifying and understanding the phenomena that may lead some successful firms to decline are relevant issues to managers and organizational leaders who can, in recognizing loss of competitive advantage factors, build other strategies for action.

Keywords: Danny Miller; The Icarus Paradox; Business Decline; Bibliometric Study.

\section{Cite it like this:}

Cunha Filho, M., Herrero, E., Mello, C., \& Vidal, D. (2019). Ascensão e Declínio De Empresas De Sucesso: estudo bibliométrico sobre o paradoxo de Ícaro. Revista Ibero-Americana de Estratégia, 18(1). 139-154. https://doi.org/10.5585/ijsm.v18i1.2741

\footnotetext{
${ }^{1}$ Universidade Nove de Julho-UNINOVE, São Paulo, Brasil. Email: mlassance@terra.com.br Orcid Id: https://orcid.org/0000-0003-4290-3147

${ }^{2}$ Universidade Nove de Julho-UNINOVE, São Paulo, Brasil. Email: liaherrero@gmail.com Orcid Id: https://orcid.org/0000-0002-4515-7242

${ }^{3}$ Universidade Nove de Julho-UNINOVE, São Paulo, Brasil. Email: claudiacmmello@ gmail.com

${ }^{4}$ Universidade Nove de Julho-UNINOVE, São Paulo, Brasil. Email: douglasvidal@ gmail.com Orcid Id: https://orcid.org/0000-0001-5406-5369
} 


\section{ASCENSÃO E DECLÍNIO DE EMPRESAS DE SUCESSO: ESTUDO BIBLIOMÉTRICO SOBRE O PARADOXO DE ÍCARO}

\section{Resumo}

Objetivo do estudo: Neste estudo, nosso objetivo foi examinar a extensão de artigos publicados com citações ao livro de Danny Miller (1990), O Paradoxo de Ícaro, e identificar as frentes de pesquisa atuais que usam a obra com referência.

Metodologia/abordagem: Este é um estudo bibliométrico com a técnica de pareamento bibliográfico.

Originalidade/Relevância: A identificação e compreensão dos fenômenos que podem levar algumas empresas de sucesso ao insucesso e de estudos que identifiquem e sugiram reflexões sobre o assunto encontra relevância para a pesquisa nas áreas das ciências sociais, especialmente no campo da estratégia organizacional.

Principais resultados: Identificamos cinco fatores que englobam os principais artigos que referenciam o livro de Miller (1990). Os temas liderança transformadora e ambiente, vantagens competitivas, recursos, processos de mudanças e percepção de oportunidades mostram as principais correntes de pesquisa que consideram e referenciam os achados de Danny Miller. Esses fatores estão voltados à pesquisa de estratégias organizacionais para a preservação da vantagem competitiva, com identificação de problemas que podem levar as empresas ao declínio.

Contribuições teóricas/metodológicas: Em nosso estudo, há uma descrição prática e detalhada do método bibliométrico com análise de citações e pareamento, utilizando a Análise Fatorial Exploratória como ferramenta para as análises. Ainda, esperamos que a descrição dos fatores possa orientar futuros pesquisadores na verificação das tendências de pesquisa sobre o tema abordado.

Contribuições sociais/para a gestão: A identificação e compreensão dos fenômenos que podem levar algumas empresas de sucesso ao declínio são relevantes para os gerentes e líderes organizacionais que podem, ao reconhecer fatores de perda de vantagem competitiva, elaborar outras estratégias de ação.

Palavras-chave: Danny Miller; Paradoxo de Ícaro; Declínio das empresas; Estudo bibliométrico.

\section{ASCENSIÓN Y DISMINUCIÓN DE EMPRESAS EXITOSAS: UN ESTUDIO BIBLIOMÉTRICO SOBRE LA PARADOJA DE ICARUS}

\section{Resumèn}

Objetivo del estudio: En este estudio, nuestro objetivo fue examinar la extensión de artículos publicados con citas del libro de Danny Miller (1990), El Paradojo de Ícaro, e identificar los frentes de investigación actuales que usan la obra con referencia.

Metodología / enfoque: Este es un estudio bibliométrico con la técnica de apareamiento bibliográfico.

Originalidad / Relevancia: La identificación y comprensión de los fenómenos que pueden llevar a algunas empresas de éxito al fracaso y de estudios que identifiquen y sugieran reflexiones sobre el tema, encuentran relevancia para la investigación en las áreas de las ciencias sociales, especialmente en el campo de la estrategia organizacional.

Principales resultados: Identificamos cinco factores que engloban los principales artículos que hacen referencia al libro de Miller (1990). Los temas de dirección transformadora y ambiente, ventajas competitivas, recursos, procesos de cambio y percepción de oportunidades muestran las principales corrientes de investigación que consideran y hacen referencia a los hallazgos de Danny Miller. Estos factores están 
Ascensão e declínio de empresas de sucesso: estudo bibliométrico sobre o paradoxo de Ícaro

orientados a la investigación de estrategias organizacionales para la preservación de la ventaja competitiva, con identificación de los problemas que pueden llevar a las empresas a la declinación.

Contribuciones teóricas / metodológicas: En nuestro estudio, hay una descripción práctica y detallada del método bibliométrico con análisis de citas y apareamiento, utilizando el Análisis Factorial Exploratorio como herramienta para los análisis. Todavía, esperamos que la descripción de los factores pueda orientar a futuros investigadores en la verificación de las tendencias de investigación sobre el tema abordado.

Contribuciones sociales / para la gestión: La identificación y comprensión de los fenómenos que pueden llevar a algunas empresas de éxito a la declinación son relevantes para los gerentes y líderes organizacionales que, al reconocer los factores de pérdida de ventaja competitiva, elaboran otras estrategias de acción.

Palabras clave: Danny Miller; Paradoja de Ícaro; Disminución De Las Empresas; Estudio Bibliométrico.

\section{INTRODUÇÃO}

Em 1990, Dany Miller lançou sua mais citada obra, O Paradoxo de Ícaro. No livro, Miller usou o mito do jovem que voou alto demais com asas feitas de penas e cera de abelhas, tendo-as derretidas pelo sol, para abordar a ascensão e declínio de grandes e lucrativas empresas. O maior poder de Ícaro estava nas asas bem construídas e robustas, mas que não poderiam suportar o calor e a proximidade do sol por muito tempo. Assim também acontece com algumas empresas bem fundamentadas e robustas: o paradoxo ocorre porque, às vezes, os seus maiores ativos as levam para a morte (Miller, 1996).

Dany Miller (1996) aponta a ironia do paradoxo, pois muitas empresas de sucesso parecem propensas a confiar demais nas principais estratégias que as conduziram, em primeiro lugar, ao sucesso. Algumas dessas estratégias estão relacionadas ao foco no negócio ou ao mercado, à confiança da liderança e a culturas galvanizadas. Esses elementos, e principalmente o conjunto deles, se excedidos em certos ambientes, podem levar ao insucesso, ao declínio e à falência (Miller, 1990). Dessa forma, empresas robustas podem se envolver em momentos confusos por meio desses excessos, políticas ineficazes e eventos insistentes, os quais as conduzem a um caminho de diminuição das vendas, encolhimento de lucros e encerramento de suas operações.

Muitos pesquisadores, principalmente nas áreas da administração, buscam compreender as razões pelas quais as empresas passam de organizações muito bem sucedidas para a falência e fechamento de portas (Holan \& Phillips, 2004; Boal \& Schultz, 2007). Há também muitos estudos em que a identificação desses riscos aponta para a criação de estratégias organizacionais específicas, na tentativa de se compreenderem dos fenômenos relacionados às organizações e à manutenção de suas vantagens competitivas (por exemplo: Judge \& Douglas, 1998; Shamsie \& Mannor, 2013).

Neste artigo, procuramos identificar as principais pesquisas em estratégia organizacional que citam $\mathrm{O}$ Paradoxo de Ícaro, de 1990, com a intenção de entender os caminhos que os principais autores sobre estratégia trilharam, quais suas principais dimensões de categorização e em que assuntos a obra de Miller foi usada como elemento de exemplificação ou teorização. Para examinar as dimensões e teorias contidas nos estudos que abordam Miller (1990) e buscar a compreensão das tendências de estudos futuros, conduzimos um estudo bibliométrico. Neste estudo, levantamos as citações e o pareamento bibliográfico, métodos que têm sido bastante usados na literatura científica nas últimas cinco décadas (Vogel \& Güttel, 2013).

Em seguida a esta introdução, apresentamos uma breve resenha do livro O Paradoxo de Ícaro, visando contextualizar o leitor sobre os principais assuntos abordados por Miller (1990) em sua obra mais citada. $\mathrm{Na}$ seção 3, estão descritos os procedimentos metodológicos deste estudo, seguidos pelos resultados, na seção 4 e descrição dos fatores, na seção 5. Concluindo este artigo, há uma seção de discussões e considerações finais.

\section{BREVE RESENHA DO LIVRO “O PARADOXO DE ÍCARO. COMO EMPRESAS EXCEPCIONAIS PROVOCAM SUA PRÓPRIA QUEDA"}

Danny Miller cunhou o paradoxo do mito grego de Ícaro na área de estratégia organizacional como analogia para empresas que experimentaram o sucesso por algum tempo, antes de encontrarem seu declínio. Como o jovem do mito, que voou perto 
demais do sol por estar inebriado com a própria capacidade de voar, Miller (1990) propôs que as empresas também são passíveis de fracasso, caso confiem demais em suas capacidades iniciais. Portanto, o principal paradoxo do sucesso se encontra na excessiva confiança de que ele será eterno.

O autor identifica as ações estratégicas dessas empresas, as quais foram inicialmente os fatores responsáveis pelo sucesso e ganhos acima da média mercadológica, mas que se modificaram durante a trajetória. Atenção produtiva ao detalhe pode se tornar uma obsessão pelas minúcias, inovação recompensadora pode iniciar um processo de invenções gratuitas e o crescimento comedido tem potencial de ser uma expansão desenfreada. Como exemplos, o autor fala de ações de marketing que viram propagandas sem apelo e de uma engenharia razoável que começa a produzir designs sem qualidade. Na contramão dessas ações, em empresas que faliram, outras atividades pouco valorizadas no início, mas que se mostrariam fundamentais para a manutenção das estratégias mais tarde, foram extintas.

Para explicar seu paradoxo, Miller (1990) descreve o declínio de grandes organizações, a partir de trajetórias organizacionais que foram iniciadas como estratégias que as levaram ao sucesso inicialmente. São elas: a) o foco excessivo; b) a tomada de risco sem equilíbrio; c) a invenção sem propósitos; e d) a desconexão estratégica. Assim, a empresa do estilo "artesão", que se preocupa com a qualidade e o detalhe, na trajetória, passa a ser a faztudo, com controle excessivo e desnecessário do que seria micro. As empresas gerenciadas pelos tipos "construtores", que são produtores criativos e planejadores, tornam-se lideradas por imperialistas que esbanjam ordens sobre o que não entendem. $\mathrm{Na}$ trajetória do "inventor", as empresas transformam pioneirismo inovador em escapismo utópico, criando invenções sem propósito. Finalmente, o perfil de "bom vendedor" inicial das empresas pode se tornar fonte de ações burocráticas de estratégias desconexas. A Tabela 1 sintetiza estas estratégias e trajetórias.

Tabela 1. Trajetória de declínio de organizações.

$\begin{array}{llll}\text { ARTESÃO } & \text { CONSTRUTOR } & \text { PIONEIRO } & \text { VENDEDOR }\end{array}$

\begin{tabular}{ccccc}
\hline $\begin{array}{c}\text { Orientação } \\
\text { estratégica } \\
\text { inicial }\end{array}$ & $\begin{array}{c}\text { Liderança } \\
\text { pela qualidade }\end{array}$ & $\begin{array}{c}\text { Expansão } \\
\text { Diversificação } \\
\text { Aquisição }\end{array}$ & $\begin{array}{c}\text { Inovação } \\
\text { Diferenciação }\end{array}$ & $\begin{array}{c}\text { Diferenciação } \\
\text { por marketing }\end{array}$ \\
\hline $\begin{array}{c}\text { Objetivo } \\
\text { principal }\end{array}$ & Qualidade & Crescimento & $\begin{array}{c}\text { Progresso } \\
\text { técnico }\end{array}$ & $\begin{array}{c}\text { Participação } \\
\text { de mercado }\end{array}$ \\
\hline
\end{tabular}

\begin{tabular}{ccccc}
\hline $\begin{array}{c}\text { Departamentos } \\
\text { dominantes }\end{array}$ & $\begin{array}{c}\text { Operações } \\
\text { Produção } \\
\text { Engenharia }\end{array}$ & $\begin{array}{c}\text { Planejamento } \\
\text { Finanças }\end{array}$ & P\&D & Marketing \\
\hline Estrutura & $\begin{array}{c}\text { Burocracia } \\
\text { Controles }\end{array}$ & $\begin{array}{c}\text { Centros de lucro } \\
\text { divisionais }\end{array}$ & $\begin{array}{c}\text { Orgânica } \\
\text { Flexível }\end{array}$ & $\begin{array}{c}\text { Divisional } \\
\text { Burocracia }\end{array}$ \\
\hline
\end{tabular}

\begin{tabular}{lccccc}
\hline Trajetória & $\begin{array}{c}\text { Foco } \\
\text { excessivo }\end{array}$ & $\begin{array}{c}\text { Risco } \\
\text { equilíbrio }\end{array}$ & sem & $\begin{array}{c}\text { Invenção } \\
\text { propósito }\end{array}$ & $\begin{array}{c}\text { Desconexão } \\
\text { estratégica }\end{array}$ \\
\hline Destino & $\begin{array}{c}\text { FAZ-TUDO } \\
\text { (tinkerer })\end{array}$ & $\begin{array}{c}\text { IMPERIALISTA } \\
\text { (imperialist })\end{array}$ & $\begin{array}{c}\text { ESCAPISTA } \\
(\text { escapist })\end{array}$ & $\begin{array}{c}\text { ANDARILHO } \\
\text { (drifter })\end{array}$
\end{tabular}

Fonte: adaptada de Miller (1990).

Com base nos casos de sucesso de empresas envolvidas nas trajetórias mencionadas, Miller (1990) consolida algumas sugestões de práticas e ações gerenciais que evitaram ou reverteram seu declínio. Para as empresas com foco excessivo em suas inovações, sem observação de conexão com o 
Ascensão e declínio de empresas de sucesso: estudo bibliométrico sobre o paradoxo de Ícaro

mercado, algumas propostas a serem implementadas são a remoção da rigidez estrutural, maior foco no mercado e competidores, diversificação de produtos e serviços obsoletos e agressividade competitiva. No caso das tomadoras de risco não balanceado com orientação estratégica expansionista, entre as ações mais efetivas, encontra-se o fortalecimento de seu conjunto de talentos em P\&D, marketing e produção, aliado a um foco maior nas competências de suas unidades de negócio e complementaridades entre suas divisões.

Para os pioneiros cuja trajetória levou ao escapismo sem fundamento, a principal ação é a redução do ímpeto inventivo apenas pela invenção propriamente dita. Maior proximidade entre áreas de marketing, produção e finanças com os cientistas para regular entusiasmo excessivo, além de colaboração entre inventores e implementadores, são ações que levaram algumas empresas a reverterem esta tendência escapista. Por fim, empresas de sucesso com reconhecida competência em vendas podem evitar estratégias desconexas com melhorias de qualidade, coordenação e controle e com uma estratégia geral e metas realistas comunicadas efetivamente em todos os níveis organizacionais.

Ao estudar casos de empresas como Polaroid, Texas Instruments, Chysler, General Motors, Apple Computers, Sears, Caterpillar Tractor, entre outras, Danny Miller foi capaz de entender e identificar que os maiores ativos de uma empresa, se percorrerem trajetórias de excesso ou estagnação, podem se tornar suas causas de morte. O paradoxo de İ́caro é esse: que muitas empresas e gestores são confiantes e complacentes demais, descuidando dos hábitos ruins. Assim, é interessante para as empresas perceberem que as mesmas causas de seu sucesso podem preceder os motivos de seus declínios.

\section{PROCEDIMENTOS METODOLÓGICOS}

Estudos bibliométricos são usados para examinar cientificamente como disciplinas, campos, especialidades e artigos se relacionam uns com os outros. Diferem das revisões de literatura, pois empregam abordagem quantitativa para descrição, avaliação e monitoramento das pesquisas publicadas (Zupic \& Čater, 2015). Entre os métodos usados em estudos bibliométricos, a análise de cocitação e o pareamento bibliográfico têm dominado a literatura científica nas últimas cinco décadas (Vogel \& Güttel, 2013).

A utilidade desses métodos para os pesquisadores é fundamentada na premissa de similaridade entre dois artigos cocitados ou pareados. Cocitação é definida como a frequência na qual dois estudos são citados simultaneamente por um terceiro estudo (Small, 1973). Quanto mais dois estudos são citados simultaneamente por outros, maior a probabilidade de que seu conteúdo seja similar. Já o pareamento usa o número de referências compartilhadas por dois estudos diferentes como uma medida de similaridade entre eles (Kessler, 1963). A diferença entre os dois métodos tem importantes implicações. Embora a premissa da similaridade seja a mesma, estudos indicam que os resultados apresentados podem ser consideravelmente diferentes daqueles pretendidos pelo pesquisador, caso o método escolhido não seja adequado (Jarneving, 2005).

O foco do pareamento bibliográfico reside nos documentos que citam outros documentos, ao invés de manter o foco nos documentos que são citados, como no caso da análise de cocitação. Dessa forma, estudos atuais indicam o pareamento como o método mais representativo, quando se busca compreender as frentes de pesquisa em andamento e os campos emergentes de estudo (Boyack \& Klavans, 2010). A análise de cocitação, por sua vez, apresenta maior vantagem quando se pretende mapear os fundamentos teóricos de um campo particular (Vogel \& Güttel, 2013).

Considerando nosso objetivo neste estudo, o de buscar as frentes de pesquisa atuais que utilizaram o livro O Paradoxo de Ícaro, de Danny Miller (1990) como referência, optamos pelo pareamento bibliográfico como método. Utilizamos a técnica de análise fatorial exploratória (AFE) com análise de componentes principais como forma de consolidação das informações dos diversos artigos em um número menor de fatores sem perda de informação e sem necessidade antecipada de estabelecimento de relações teóricas entre eles (Hair, Black, Babin, Anderson, \& Tatham, 2006; Conway \& Huffcutt 2003). Optamos também pela análise e visualização de rede por meio do software UCINET (Borgatti, Everett, \& Freeman, 2002). O algoritmo prepara a rede para visualização com base na distância entre os artigos (nós) calculada pelo número de ligações por pareamento (força da conexão) entre eles.

\section{RESULTADOS}

Para a busca e extração dos artigos, usamos a base de dados de referências citadas do Web of Science (WoS - Clarivate Analytics) com o booleano ("the icarus paradox" OR "icarus paradox"). Dos 126 documentos obtidos inicialmente, filtramos somente os artigos publicados das áreas de gestão e negócios (management / business) obtendo nossa base final com 86 artigos. Entre estes documentos, 
selecionamos para extração os campos: título, autores, periódico, referências citadas, resumo, palavras-chave e número de citações.

Dos 86 estudos obtidos, 73 (85\%) apresentam fator de impacto (FI) maior ou igual a 1,4 , com 57 $(66 \%)$ deles acima de 3 , conforme dados atualizados em dezembro de 2018. A qualidade e relevância dos periódicos demonstra a importância dos temas tratados para as organizações e sua correspondente ligação às preocupações determinadas no livro Paradoxo de Ícaro. A Tabela 2 mostra os periódicos com FI maior ou igual a 3 e a quantidade de artigos publicados, conforme base extraída.

Tabela 2. Periódicos

\begin{tabular}{lcc}
\hline \hline Periódico & $\begin{array}{c}\text { Artigos } \\
\text { publicados }\end{array}$ & $\begin{array}{c}\text { Fator } \\
\text { de impacto } \\
\text { - periódico }\end{array}$ \\
\hline Academy of Management Review & 4 & 8,86 \\
Journal of The Academy of Marketing Science & 1 & 8,49 \\
Journal of Management & 4 & 8,08 \\
Academy of Management Journal & 4 & 6,70 \\
Journal of Business Venturing & 4 & 6,00 \\
Administrative Science Quarterly & 3 & 5,88 \\
Strategic Management Journal & 9 & 5,48 \\
Journal of Management Studies & 5 & 5,33 \\
Entrepreneurship Theory And Practice & 4 & 5,32 \\
Journal of Information Technology & 1 & 4,54 \\
Journal of Organizational Behavior & 1 & 4,23 \\
Journal of International Marketing & 1 & 3,60 \\
Management Science & 1 & 3,54 \\
Strategic Entrepreneurship Journal & 2 & 3,49 \\
Leadership Quarterly & 2 & 3,31 \\
California Management Review & 1 & 3,30 \\
Journal of Small Business Management & 1 & 3,25 \\
Long Range Planning & 1 & 3,22 \\
Public Management Review & 1 & 3,15 \\
Organization Studies & 3 & 3,13 \\
Human Relations & 1 & 3,04 \\
Organization Science & 3 & 3,03 \\
\cline { 2 - 3 } & &
\end{tabular}

Fonte: Elaborada pelos autores.

Carregamos os dados obtidos do WoS no aplicativo Microsoft Office-EXCEL 2013, para preparação e limpeza dos arquivos a serem usados para carga nos programas utilizados como apoio para nosso estudo. Agrupamos os artigos por ano de publicação e título para inspeção e correção dos artigos idênticos referenciados de forma diferente. A planilha preparada foi convertida em arquivo texto observando os marcadores especiais de campo para carga nos softwares de análise.

Optamos pela utilização do software R com o pacote bibliometrix (Aria \& Cuccurullo 2017; Efrain-García \& García-Zorita, 2016), para análise descritiva e preparação da matriz de pareamento. Enquanto os elementos (Eij $\mid \mathrm{i} \neq \mathrm{j}$ ) fora da diagonal na matriz gerada pelo $\mathrm{R}$ indicam a quantidade de documentos citados simultaneamente pelos artigos i e $\mathrm{j}$, os elementos da diagonal (Eij $\mid \mathrm{i}=\mathrm{j}$ ) mostram a quantidade total de documentos citados pelo artigo i $(=\mathrm{j})$. Esses elementos da diagonal devem ser tratados antes da entrada de dados para a AFE. Podem ser tratados como missing values (Vogel \& Güttel, 2013) ou, como em nosso caso, atribuímos o valor zero para adequação ao software usado para análise fatorial exploratória. A Tabela 3 resume as principais informações sobre a base extraída. 
Tabela 3. Informações sobre a base de dados extraída.

\begin{tabular}{ll}
\hline Documentos - base & 86 \\
Periódicos & 46 \\
Palavras-chave & 153 \\
Período & $1991-2018$ \\
Média de citações por documento & 95,69 \\
Quantidade total de autores & 156 \\
No $^{\circ}$ documentos com apenas 1 autor & 15 \\
Autores - documentos com 1 autor & 11 \\
Média de autores por documento & 1,81 \\
Média de coautores por documento & 2,24 \\
\hline
\end{tabular}

Fonte: elaborada pelos autores.

Para a análise fatorial exploratória, com auxílio do software IBM SPSS, usamos a análise de componentes principais com normalização Kaiser e rotação ortogonal VARIMAX. Observamos a adequação dos dados para este tipo de análise, obtendo resultado de teste $\mathrm{KMO}$ maior que 0,5 e teste de esfericidade de Bartlett com significância menor que 0,05 (Hair et al., 2006). Para certificação da validade dos resultados obtidos, verificamos também comunalidade maior que 0,5 entre os artigos e confiabilidade da escala maior que 0,7 , com o cálculo do alfa de Cronbach (Hair et al., 2006). A Tabela 4 resume os resultados obtidos para adequação de dados, validade e confiabilidade.

Tabela 4. Adequação, validade e confiabilidade

\begin{tabular}{lc}
\hline \hline Adequação da amostra - KMO & 0,523 \\
Esfericidade de Bartlett's - significância & 0,000 \\
Comunalidade mínima entre artigos & 0,629 \\
Alfa de Cronbach & 0,943 \\
\hline Fonte: elaborada pelos autores.
\end{tabular}

$\mathrm{Na}$ primeira rodada da AFE com rotação VARIMAX, o programa apresentou 20 fatores para extração, representando mais de $77 \%$ da variância explicada. Observando o gráfico screeplot gerado e a composição dos artigos por fator, optamos por fixar o número total de fatores em cinco.

A variância explicada destes cinco fatores excedeu $50 \%$, sem maior comprometimento da estrutura para análise. Para composição dos fatores, usamos apenas os artigos que apresentaram carga fatorial maior que 0,4 (McCain, 1990). Para os artigos que apresentaram cargas cruzadas entre fatores, foi escolhido o fator no qual essa carga fatorial fosse maior (Vogel \& Güttel, 2013).

Como complemento da AFE, usamos a análise de rede, com apoio do software UCINET 6. Com o propósito de facilitar a visualização, escolhemos os artigos (nós) com força de conexão superior a 7 (tie strenght $>7$ ) e grau mínimo 2 (degree $\geq 2$ ). Ou seja, artigos cuja quantidade total de acoplamentos (conexões) fosse superior a $7 \mathrm{com}$, no mínimo, dois outros artigos. A Figura 1 mostra a rede agrupada, conforme a distância entre os nós medida pela força de conexão entre eles. A representação gráfica dos nós indica o fator ao qual cada nó pertence. 
Figura 1. Rede de pareamento.

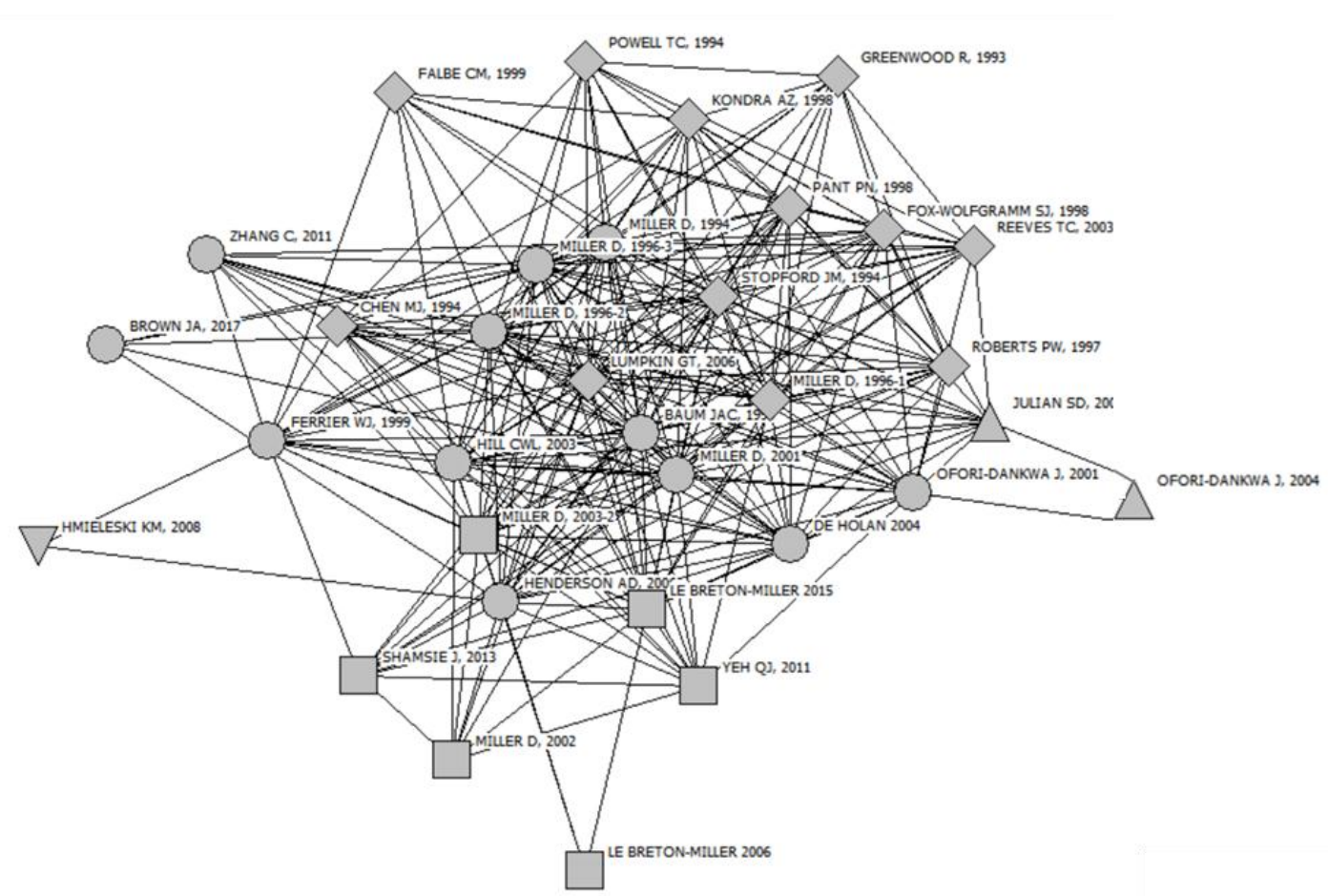

Fonte: elaborada pelos autores.

Em adição à visualização da rede de pareamento, optamos por apresentar duas medidas relevantes relacionadas aos fatores definidos na AFE: densidade e índice E-I. A densidade é calculada pela razão entre a totalidade das forças de conexão existentes no fator e a quantidade total de possíveis conexões com força de conexão igual a 1. A densidade pode ser usada como uma medida de distinção do fator, quando comparada à densidade total da rede. O índice E-I é calculado como o número de conexões externas ao fator menos o número de conexões internas, dividido pelo número total de conexões da rede. Este índice é uma medida de dominância entre o número de conexões externas ao fator e o número de conexões internas (Krackhardt \& Stern, 1988). Assim, quanto menor o índice maior a relevância das conexões internas ao fator em relação às externas. A Tabela 5 mostra a relação de fatores extraídos, consolidando as informações obtidas na AFE com o SPSS e na análise de rede com o UCINET.

Tabela 5. Rede de pareamento: análise fatorial e medicões de rede

\begin{tabular}{|c|c|c|c|c|c|c|}
\hline \multirow[t]{2}{*}{ Fator } & \multirow[t]{2}{*}{ Símbolo } & \multirow[t]{2}{*}{ Artigos } & \multicolumn{2}{|c|}{ Análise fatorial } & \multicolumn{2}{|c|}{ Análise de rede } \\
\hline & & & Eigenvalue & $\begin{array}{c}\text { Variância } \\
\text { Explicada (\%) }\end{array}$ & Densidade $^{1}$ & $\begin{array}{l}\text { Índice } \\
\text { E-I }\end{array}$ \\
\hline 1 & & 26 & 13,217 & 16,731 & 2,966 & 0,427 \\
\hline 2 & & 18 & 10,947 & 13,857 & 4,719 & 0,600 \\
\hline 3 & & 9 & 6,182 & 7,825 & 3,861 & 0,804 \\
\hline 4 & $\triangle$ & 9 & 5,534 & 7,005 & 1,694 & 0,804 \\
\hline 5 & & 5 & 3,883 & 4,916 & 1,200 & 0,902 \\
\hline
\end{tabular}

1 Densidade da rede completa: 1,869

Fonte: elaborada pelos autores 
Entre estes grupos (fatores) com propósitos similares que emergiram em nosso pareamento bibliográfico, é importante considerar a presença de artigos com carga cruzada indicando variância explicada entre grupos distintos estes estudos. Este ponto é mais marcante entre os Fatores 1 e 2, tendo como exemplos os trabalhos de Chen e Miller (1994) e o de McKelvey (1997), em que ambos buscam equilibrar sua argumentação com referências às ações competitivas e ao papel da liderança. Como exemplo de carga cruzada entre os Fatores 2 e 3, está o trabalho de Le Breton e Miller (2015), que aborda a vulnerabilidade dos recursos na sustentação de vantagem competitiva. Estas cargas cruzadas representam manifestações de inter-relacionamento entre os fatores observados como resultado do estudo, evidenciando assim, alguma natureza similar entre seus propósitos de pesquisa.

\section{FATORES}

Após as análises e identificação dos fatores, passamos ao estudo dos artigos contidos em cada um, para denominação e descrição das tendências de cada dimensão. O Fator 1 engloba 26 artigos com cargas fatoriais que variam entre 0,409 e 0,804 . Denominamos o fator como "Liderança transformacional e ambiente" e, no geral, os estudos mostram que as qualidades que as empresas deveriam buscar em seus líderes não estão totalmente associadas apenas à qualidade total da gerência (Stopford \& Baden-Fuller, 1994; Lamothe \& Dufour, 2007).

As empresas deveriam se importar, principalmente, com outros elementos de sustentabilidade de vantagens estratégicas, como adaptação de recursos da liderança para resposta aos ambientes (Judge \& Douglas, 1998). Os ambientes internos (Kondra \& Hinings, 1998), os externos (Reeves, Duncan, \& Ginter, 2003) e os de controle social (Pant \& Lachman, 1998) necessitam de gestores e equipes que percebam as mudanças e adaptem-se a elas (Lumpkin \& Dess, 2006).

Pawar e Eastman (1997) apontam quatro fatores contextuais que afetam a receptividade dos empregados à nova liderança transformacional que, se não sanados, comprometem a sustentação do sucesso da empresa. Greenwood e Hinings (1993) usam arquétipos de liderança para explicar o paradoxo encontrado entre a necessidade de qualidade da liderança que pretende trazer mudança e os aspectos negativos associados ao excesso de tal característica, tópico encontrado também em
Langley (1995), Moulton, Thomas e Pruett, (1996) e Roberts e Greenwood (1997).

Os papéis da cognição e das habilidades dos líderes organizacionais como principais expoentes da manutenção do sucesso na empresa são abordados por alguns autores presentes no fator 1 (Meyer, 1991; Schreuder, 1993; Powell, 1994; Fox-Wolfgramm, Boal, \& Hunt, 1998). Mintzberg (1991) coloca a questão do aprendizado organizacional, do papel do líder e seu poder de processos cognitivos para adaptar-se às mudanças em complementaridade à característica de planejamento que deve estar inerente à função.

De modo geral, as empresas necessitam estar atentas aos líderes em suas relações com os empregados acima de tudo. A formação estratégica das equipes cabe, em grande parcela, aos líderes (Block, Kohn, K, Miller, \& Ullrich, 2015). Porém, se não há fórmulas precisas que garantam o sucesso perene das empresas (Chen \& Miller, 1994), há que se cuidar que as estratégias focais não sejam ambiciosas demais Valentin, 1994; Miller, 1996; Miller \& Sardais, 2015).

O Fator 2, denominado "Ação para vantagem competitiva", contém 18 artigos e as cargas fatoriais se estendem de 0,432 até 0,736. Nessa dimensão, há indicativos que a inércia das corporações, muitas vezes originadas na segurança aparente do sucesso do momento, culmina em declínio das vendas e financeiro (Hill \& Rothaermel, 2003; Jayachandran \& Varadarajan, 2006; Holan \& Phillips, 2004; Lampel \& Jha, 2017). Este fator aponta diretamente para a abordagem de Miller (1990), ao estabelecer que o paradoxo da garantia ilusória de que o sucesso é perene e infinito faz com que empresas e gestores se acomodem (Zhang, Song, \& Qu, 2011) e deixem de traçar estratégias novas e atuais para manutenção da vantagem competitiva (Henderson, Miller, \& Hambrick, 2006; Walters, Kroll, \& Wright, 2007; Brown, Anderson, Salas, \& Ward, 2017).

Dentro dessas perspectivas de necessidade de atualização, ação, exploration e exploitation constantes, o aprendizado organizacional para a criação e recriação de estratégias precisa ocorrer rotineiramente (Ferrier, Smith, \& Grimm, 1999; Garcia, Calantone, \& Levine, 2003; Yukl, 2009). A aplicação do aprendizado precisa ocorrer em ações longitudinais e enquanto a empresa estiver atuando (Baum \& Berta, 1999; Ofori-Dankwa \& Julian, 2001; Boal \& Schultz, 2007).

O próprio Danny Miller aparece, no Fator 2, como autor principal de três artigos, nos quais se 
autocita. Neles, há apontamentos sobre a manutenção de competitividade por meio de recursos e verificação de ambientes externos, sobre a inércia como ocasionadora de insucesso organizacional e aplicação de aprendizado através do tempo para fins de vantagens estratégicas (Miller \& Chen, 1994; Miller \& Shamsie, 1996; Miller \& Shamsie, 2001).

O Fator 3, chamado de "Gestão de recursos para sustentabilidade do sucesso" é composto por nove artigos (cargas fatoriais entre 0,508 e 0,825), sendo que seis discorrem sobre a visão baseada em recursos como base estratégica das empresas, e os outros três, sobre gestão de recursos. Dessa forma, os aspectos da visão baseada em recursos e a gestão são tratados de diversas maneiras. Miller (2003) e Le BretonMiller e Miller $(2006,2015)$ discutem as capacidades que resultarão em simetria e assimetria de recursos para gerar vantagem competitiva sustentável. Os executivos precisam desenvolver capacidades, motivando seus parceiros ao mesmo para a superação das assimetrias (Miller, Eisenstat, \& Foote, 2002).

Dentro da mesma abordagem de visão baseada em recursos, Yeh e Fang (2011) fazem o elo com o ciclo de vida organizacional, no qual o sucesso passado da empresa pode cegá-la de tal forma que comprometa as oportunidades futuras e ameaças presentes. A gestão de recursos como elemento crítico na dimensão da conversão do conhecimento tácito nas rotinas também é apontada (Johnson, Yip, \& Hensmans, 2012; Shamsie \& Mannor, 2013), inclusive por autores que buscam soluções de busca e criação de valor para sustentação do desempenho da empresa dentro de mercados em mudança (Covin, Garrett, Kuratko, \& Shepherd, 2015). No ambiente do e-business, Ramsey, Ibbotson, Bell e Mccole (2005) indicam que a inovação tecnológica é um dos motores de mudança da economia e adaptação dos recursos e determinação dos limites tecnológicos e culturais que levem a uma força ou fraqueza.

Nomeamos o Fator 4 como "Processos de Mudança". Este fator é composto por nove artigos, com cargas fatoriais que variam de 0,440 até 0,654. Mudanças estratégicas são partes importantes da configuração da empresa em sua linha do tempo, para manter as vantagens de mercado. Miller e Le BretonMiller (2017) destacam que essas mudanças podem ser dinâmicas ou estáveis, de acordo com suas estratégias. Assim, o grande desafio das empresas é considerar a mudança e não o equilíbrio como princípio da vida (Calori, Baden-Fuller, \& Hunt, 2000), pois pode haver uma tendência natural da empresa, depois de certo tempo, para relaxar ou até mesmo entrar em "sono profundo", se o mercado não for dinâmico.

É improvável que a mesma estratégia usada mais de duas vezes tenha exatamente os mesmos resultados organizacionais, pois ações que tiveram sucesso no passado podem falhar diante de um novo cenário (Thietart, \& Forgues, 1997; Colville, Waterman, \& Weick, 1999).

Também é preciso levar em consideração as condições internas da empresa, como, por exemplo, a motivação dos colaboradores em relação às políticas salariais, de comissão, que tem impacto e podem levar a mudanças que determinam o sucesso ou fracasso de uma empresa (Ofori-Dankwa \& Julian, 2004). Assim ocorre ainda com o processo de decisão, com a insegurança ou excesso de confiança dos gestores, o que pode causar a morte da empresa (Kotlyar \& Karakowsky 2007). Desta forma, a natureza da mudança representa uma transformação na empresa, que pode ser radical ou incremental (Bennett, McCracken, \& O'Kane, 2018), pode ser direcionada pelo gestor (David \& Strang, 2006) e que, quando relacionada com a falta recursos, leva à menor capacidade de resposta e, consequentemente, menor reação para mudanças (Julian \& OforiDankwa, 2008).

O Fator 5, nomeado de "Percepção de oportunidades", está representado na amostra por cinco artigos, com cargas entre 0,412 e 0,584 . Todos os artigos abordam como os gestores das empresas percebem o ambiente e suas oportunidades, inclusive quando são responsáveis pela contratação de outros recursos humanos. No artigo de Krueger Jr. (1998), o autor aponta que as oportunidades devem ser encontradas e não inventadas, tendo-se, assim, a necessidade de uma equipe em sintonia para a identificação de todas as pistas do ambiente.

$\mathrm{Na}$ mesma linha de importância da criação de uma equipe que identifique as boas oportunidades de negócios, e mesmo aquelas que são ruins, devem ser levadas em conta as características pessoais. Os processos cognitivos e o foco regulatório individual são componentes a se considerar em um time organizacional que não se deixe deslumbrar por oportunidades falsas de sucesso (Krueger Jr. \& Dickson, 1994; Hmieleski \& Baron, 2008).

Os outros dois artigos do Fator 5 identificam que a confiança excessiva na certeza da percepção das oportunidades e melhores recursos pelos CEOs pode ser um fator de insucesso Greer \& Ireland, 1992), fenômenos que pode ser acentuado em empresas familiares (Miller, Steier, \& Le Breton-Miller, 2003). 
Ascensão e declínio de empresas de sucesso: estudo bibliométrico sobre o paradoxo de Ícaro

\section{DISCUSSÕES E CONSIDERAÇÕES FINAIS}

Os resultados deste estudo revelam as diversas áreas de interesse nas quais o livro Paradoxo de Ícaro foi utilizado como referência. A rede obtida da literatura, com base no compartilhamento de referências usadas, ilustra bem a densidade e interconexão entre esses estudos. Com o suporte da análise fatorial e da análise de rede foi possível detectar os principais tópicos que orientaram as pesquisas realizadas a partir da publicação da edição inicial do livro em 1990.

Em essência, o livro de Danny Miller descreve o declínio de grandes organizações bem sucedidas, a partir de quatro trajetórias (foco excessivo, risco sem equilíbrio, invenção sem propósito e desconexão estratégica), cujo ponto de partida se fundamenta nas orientações estratégicas que as levaram ao sucesso demonstrado por retornos excepcionais e acima da média de mercado. Os temas liderança transformadora, vantagens competitivas, recursos, processos de mudanças e percepção de oportunidades, associados aos fatores levantados pelo estudo, mostram as principais correntes de pesquisa que consideram e referenciam os achados de Danny Miller.

Os autores dos artigos que compõem o fator "Liderança transformacional e ambiente", buscam, em geral, avaliar o dinamismo entre definição, planejamento e execução estratégicas das empresas, como elementos de diferenciação de desempenho. Têm como componente comum a relevância dada ao papel da liderança da organização em cada uma destas etapas e suas interações. Há diferentes abordagens entre os gestores para a estratégia (Mintzberg, 1991) que podem levar a empresa a desempenho não satisfatório e eventual declínio. Um falso senso de controle e segurança nestes processos estratégicos podem provocar apatia e complacência nos gestores (Powell, 1994), afastando a empresa do estado de adaptação contínua e a levando a inércia (Schreuder, 1993).

Independente da orientação estratégica da empresa e da eventual coincidência de sua trajetória com quaisquer dos caminhos para o declínio explicitados por Danny Miller no Paradoxo de Ícaro, a atuação dos gestores como líderes transformadores pode evitar ou reverter o processo de declínio. Como exemplo, seja na flexibilização de uma estrutura organizacional rígida para as empresas com foco excessivo em inovações, ou na promoção de maior colaboração entre cientistas e implementadores no caso das pioneiras com tendência ao escapismo sem fundamento, a atuação da gestão é foco de estudo. Importante mencionar que dos 26 artigos deste fator, apenas 5 foram publicados após a década de 1990, caracterizando uma orientação de pesquisa nesta linha mais próxima à data de publicação do livro Paradoxo de Ícaro.

No grupo de artigos do fator "Ação para vantagem competitiva", destacamos a questão da aprendizagem organizacional como elemento (Miller \& Shamsie, 2001; Henderson et al., 2006) necessário para adaptação a mudanças e tomada de ações para obtenção e sustentação de vantagem competitiva. Alguns artigos mencionam CEO (chief executive officers) e alta gerência em geral como sujeitos de pesquisa (Henderson et al., 2006; Brown et al. 2017; Boal \& Schultz, 2007), avaliando a relação entre seu aprendizado contínuo, a inércia organizacional e o desempenho da empresa.

Após seu aprendizado inicial, e eventual sucesso com as ações tomadas para a competitividade, há uma acomodação desta alta liderança com a situação aparentemente estável, caracterizando um estado de inércia organizacional. Seja em qualquer das orientações estratégicas que levaram organizações a um desempenho superior à concorrência, conforme descrito no livro Paradoxo de Ícaro, esta pressuposta hipótese de que o conhecimento já adquirido com o aprendizado inicial é suficiente, pode levar as empresas a caírem nas armadilhas de seu próprio sucesso. Em movimento distinto ao fator 1, mostrando uma orientação mais atual, treze dos 18 artigos deste fator foram publicados após o ano 2000, sendo 2 deles publicados há menos de 2 anos.

Em "Gestão de recursos para sustentabilidade do sucesso", o foco é voltado aos recursos organizacionais e sua importância como elemento de diferenciação e agregação de valor ao negócio para sustentação de vantagens competitivas (Miller, 2003; Le Breton-Miller \& Miller, 2006, 2015). No caso das empresas tomadoras de risco não balanceado com orientação estratégica expansionista descrito por Miller, uma das práticas que podem evitar ou mesmo reverter seu declínio é o fortalecimento de seu conjunto de talentos em $\mathrm{P} \& \mathrm{D}$, marketing e produção. Com esta base nestes conhecimentos de difícil imitação por seus concorrentes, estas empresas expansionistas podem equilibrar suas decisões de crescimento orgânico e não orgânico conforme aumenta sua capacidade de percepção e mitigação de riscos antes não observados.

No fator 4, "Processos de Mudança", os autores apontam a mudança e não o equilíbrio ou inércia como orientação organizacional por excelência 
(Calori, Baden-Fuller, \& Hunt, 2000). Sem uma contínua revisão de seus processos volta a questão da acomodação com consequências que podem ser as indicadas no livro de Danny Miller. Revisão e mudança nos processos de melhoria de qualidade, coordenação e controle por exemplo, já demonstraram efetividade na reversão de trajetória de declínio em empresas cujo sucesso anterior com sua reconhecida competência em vendas foi arrefecido por estratégias desconexas e falta de foco.

Por fim, o grupo "Percepção de oportunidades" reforça a natureza dinâmica do mercado e a contínua atenção dos gestores organizacionais às oportunidades, sejam elas para defesa de suas posições competitivas ou mesmo para criar ou tomar mercados. Num paralelo ao mencionado no livro de Danny Miller, maior foco no mercado e competidores para percepção de oportunidades é uma das receitas que já se provaram efetivas para empresas que caíram na armadilha do foco excessivo em inovações.

Em resumo, os Fatores 1, 2 e 3, com densidades acima do valor apresentado pela rede completa e com variância total explicada próxima a 40\%, apresentam a maior relevância entre os campos de estudo que referenciam $\mathrm{O}$ Paradoxo de Ícaro. Há destaque para o Fator 1 - "Liderança transformacional e ambiente" - com mais de $30 \%$ dos artigos de nossa base inicial. Estes resultados suportam que o papel da liderança transformadora, as ações para sustentação de vantagens competitivas e a gestão de recursos são os campos que lideram a abordagem à questão do declínio organizacional em suas argumentações, conforme descrito por Danny Miller no livro.

Dada a relevância acadêmica da maioria dos artigos encontrados em nossa análise, esperamos que este estudo contribua para a continuidade de pesquisas que visam identificar e buscar soluções estratégicas organizacionais para problemas de empresas bem sucedidas que enfrentam possibilidades de declínio. De toda maneira, os fatores que identificamos e classificamos trazem insights interessantes sobre as características das empresas que, mesmo sendo consideradas como relevantes, podem, em excesso ou por tempo longo, trazer riscos à continuidade dos negócios.

As limitações que cercam esta pesquisa são inerentes aos estudos bibliométricos, como por exemplo, o viés causado pelas autocitações (Zupic \& Čater, 2015). No caso deste estudo, encontra-se também o limite da busca das palavras-chave apenas na base de dados Web of Science. Para futuros estudos, sugerimos a busca em bases diferentes, como a SCOPUS e a EBSCO, por exemplo.

Esperamos ter, de alguma forma, contribuído para a identificação das tendências de estudo da academia sobre as razões do fracasso organizacional e sobre quais ações estratégicas podem ser tomadas para evitar o declínio de empresas de sucesso.

\section{REFERÊNCIAS}

Aria, M., \& Cuccurullo, C. (2017). bibliometrix: An R-tool for comprehensive science mapping analysis. Journal of Informetrics, 11(4), 959-975.

Baum, J. A., \& Berta, W. B. (1999). Sources, dynamics, and speed: A longitudinal behaviorial simulation of interorganizational and populationlevel learning. Advances in strategic management, VOL 16-1999, 16, 155-184.

Bennett, H., McCracken, M., \& O'Kane, P. (2018). 'Engage for change' The development of a Radical Change Engagement model. Journal of General Management, 43(3), 115-125.

Block, J. H., Kohn, K., Miller, D., \& Ullrich, K. (2015). Necessity entrepreneurship and competitive strategy. Small Business Economics, 44(1), 37-54.

Boal, K. B., \& Schultz, P. L. (2007). Storytelling, time, and evolution: The role of strategic leadership in complex adaptive systems. The leadership quarterly, 18(4), 411-428.

Borgatti, S. P., Everett, M. G., \& Freeman, L. C. (2002). Ucinet for Windows: Software for social network analysis.

Boyack, K., \& Klavans, R. (2010). Co-citation analysis, bibliographic coupling, and direct citation: Which citation approach represents the research front most accurately? Journal of the American Society for Information Science and Technology, 61(12), 23892404. doi:10.1002/asi

Brown, J. A., Anderson, A., Salas, J. M., \& Ward, A. J. (2017). Do investors care about director tenure? Insights from executive cognition and social capital theories. Organization Science, 28(3), 471-494.

Calori, R., Baden-Fuller, C., \& Hunt, B. (2000). Managing change at Novotel: back to the future. Long Range Planning, 33(6), 779-804.

Chen, M. J., \& Miller, D. (1994). Competitive attack, retaliation and performance: an 
Ascensão e declínio de empresas de sucesso: estudo bibliométrico sobre o paradoxo de Ícaro

expectancy\&valence framework. Strategic Management Journal, 15(2), 85-102.

Colville, I. D., Waterman, R. H., \& Weick, K. E. (1999). Organizing and the search for excellence: Making sense of the times in theory and practice. Organization, 6(1), 129-148.

Conway, J. M., \& Huffcutt, A. I. (2003). A Review and Evaluation of Exploratory Factor Analysis Practices in Organizational Research. Organizational Research Methods, 6(2), 147-168. doi:10.1177/1094428103251541

Covin, J. G., Garrett, R. P., Kuratko, D. F., \& Shepherd, D. A. (2015). Value proposition evolution and the performance of internal corporate ventures. Journal of Business Venturing, 30(5), 749774.

David, R. J., \& Strang, D. (2006). When fashion is fleeting: Transitory collective beliefs and the dynamics of TQM consulting. Academy of Management Journal, 49(2), 215-233.

Efrain-García, P., \& García-Zorita, C. (2016). "Desktop Scientometrics": una metodología para el uso de datos procedentes de WoS mediante el programa estadístico R. Revista española de Documentación Científica, 39(1), 122.

Ferrier, W. J., Smith, K. G., \& Grimm, C. M. (1999). The role of competitive action in market share erosion and industry dethronement: A study of industry leaders and challengers. Academy of management journal, 42(4), 372-388.

Fox-Wolfgramm, S. J., Boal, K. B., \& James G.(Jerry) Hunt. (1998). Organizational adaptation to institutional change: A comparative study of firstorder change in prospector and defender banks. Administrative Science Quarterly, 87-126.

Garcia, R., Calantone, R., \& Levine, R. (2003). The role of knowledge in resource allocation to exploration versus exploitation in technologically oriented organizations. Decision sciences, 34(2), 323-349.

Greenwood, R., \& Hinings, C. R. (1993). Understanding strategic change: The contribution of archetypes. Academy of management Journal

Greer, C. R., \& Ireland, T. C. (1992). Organizational and financial correlates of a "contrarian" human resource investment strategy. Academy of Management Journal, 35(5), 956-984.

Hair, J. F., Black, W. C., Babin, B. J., Anderson, R. E., \& Tatham, R. L. (2006). Multivariate data analysis (Vol. 6).

Hambrick DC, Geletkanycz MA, Fredrickson JW (1993) Top executive commitment to the status quo: Some tests of its determinants. Strategic Management J. 14(6):401-418.

Henderson, A. D., Miller, D., \& Hambrick, D. C. (2006). How quickly do CEOs become obsolete? Industry dynamism, CEO tenure, and company performance. Strategic Management Journal, 27(5), 447-460.

Hill, C. W., \& Rothaermel, F. T. (2003). The performance of incumbent firms in the face of radical technological innovation. Academy of Management Review, 28(2), 257-274.

Hmieleski, K. M., \& Baron, R. A. (2008). Regulatory focus and new venture performance: A study of entrepreneurial opportunity exploitation under conditions of risk versus uncertainty. Strategic Entrepreneurship Journal, 2(4), 285-299.

Holan, P. M. D., \& Phillips, N. (2004). Remembrance of things past? The dynamics of organizational forgetting. Management science, 50(11), 1603-1613.

Jarneving, B. (2005). A comparison of two bibliometric methods for mapping of the research front. Scientometrics, 65(2), 245-263.

Jayachandran, S., \& Varadarajan, R. (2006). Does success diminish competitive responsiveness? Reconciling conflicting perspectives. Journal of the Academy of Marketing Science, 34(3), 284.

Johnson, G., Yip, G. S., \& Hensmans, M. (2012). Achieving successful strategic transformation. MIT Sloan Management Review, 53(3), 25.

Judge, W. Q., \& Douglas, T. J. (1998). Performance implications of incorporating natural environmental issues into the strategic planning process: an empirical assessment. Journal of management Studies, 35(2), 241-262.

Julian, S. D., \& Ofori-Dankwa, J. C. (2008). Toward an integrative cartography of two strategic issue diagnosis frameworks. Strategic Management Journal, 29(1), 93-114. 
Kessler, M. M. (1963). Bibliographic coupling between scientific papers. American documentation, 14(1), 10-25. Jarneving, B. (2005). A comparison of two bibliometric methods for mapping of the research front. Scientometrics, 65(2), 245-263.

Kondra, A. Z., \& Hinings, C. R. (1998). Organizational diversity and change in institutional theory. Organization studies, 19(5), 743-767.

Kotlyar, I., \& Karakowsky, L. (2007). The positive side of negative framing: Examining a case of overconfidence among decision makers in a hospital merger. Management Decision, 45(6), 968981.

Krackhardt, D., \& Stern, R. N. (1988). Informal networks and organizational crises: An experimental simulation. Social psychology quarterly, 123-140.

Krueger Jr, N. (1998). Encouraging the identification of environmental opportunities. Journal of Organizational Change Management, 11(2), 174-183.

\& Dickson, P. R. (1994). How believing in ourselves increases risk taking: Perceived selfefficacy and opportunity recognition. Decision Sciences, 25(3), 385-400.

Lampel, J., \& Jha, P. P. (2017). Inertia, aspirations, and response to attainment discrepancy in design contests. $R \& D$ Management, 47(4), 557569.

Lamothe, L., \& Dufour, Y. (2007). Systems of interdependency and core orchestrating themes at health care unit level: a configurational approach. Public Management Review, 9(1), 67-85.

Langley, A. (1995). Between "paralysis by analysis" and "extinction by instinct". Sloan Management Review, 36, 63-63.

Le Breton-Miller, I., \& Miller, D. (2006). Why Do Some Family Businesses Out-Compete? Governance, Long-Term Orientations, and Sustainable Capability. Entrepreneurship theory and practice, 30(6), 731-746.

(2015). The paradox of resource vulnerability: Considerations for organizational curatorship. Strategic management journal, 36(3), 397-415.
Lumpkin, G. T., \& Dess, G. G. (2006). The effect of 'simplicity'on the strategy-performance relationship: A note. Journal of Management Studies, 43(7), 1583-1604.

McCain, K. W. (1990). Mapping authors in intellectual space: A technical overview. Journal of the American society for information science, 41(6), 433-443.

Meyer, A. D. (1991). What is strategy's distinctive competence?. Journal of Management, 17(4), 821-833.

Miller, D. (1990). The Icarus paradox: How excellent organizations can bring about their own downfall. Haper Business, New York, NY.

(1992). The Icarus paradox: How exceptional companies bring about their own demise.

(1996). Configurations revisited. Strategic management journal, 17(7), 505512.

(2003). An asymmetry-based view of advantage: towards an attainable sustainability. Strategic management journal, 24(10), 961-976.

\& Chen, M. J. (1994). Sources and consequences of competitive inertia: A study of the US airline industry. Administrative science quarterly, 1-23.

Eisenstat, R., \& Foote, N. (2002). Strategy from the inside out: Building capabilitycreating organizations. California Management Review, 44(3), 37-54.

\& Le Breton-Miller, I. (2017). Underdog entrepreneurs: A model of challenge-based entrepreneurship. Entrepreneurship Theory and Practice, 41(1), 7-17.

, Eisenstat, R., \& Foote, N. (2002). Strategy from the inside out: Building capabilitycreating organizations. California Management Review, 44(3), 37-54.

\& Sardais, C. (2015). Bifurcating time: How entrepreneurs reconcile the paradoxical demands of the job. Entrepreneurship Theory and Practice, 39(3), 489-512. 
Ascensão e declínio de empresas de sucesso: estudo bibliométrico sobre o paradoxo de Ícaro

\& Shamsie, J. (1996). The resource-based view of the firm in two environments: The Hollywood film studios from 1936 to 1965 . Academy of management journal, 39(3), 519-543.

\& Shamsie, J. (2001). Learning across the life cycle: Experimentation and performance among the Hollywood studio heads. Strategic Management Journal, 22(8), 725-745.

Steier, L., \& Le Breton-Miller, I. (2003). Lost in time: Intergenerational succession, change, and failure in family business. Journal of business venturing, 18(4), 513-531.

Mintzberg, H. (1991). The effective organization: forces and forms. MIT Sloan Management Review, 32(2), 54-67.

Moulton, W. N., Thomas, H., \& Pruett, M. (1996). Business Failure Path ways: Environmental Stress and Organizational Response. Journal of Management, 22(4), 571-595.

Ofori-Dankwa, J. \& Julian, S. D. (2001). Complexifying organizational theory: Illustrations using time research. Academy of Management Review, 26(3), 415-430.

(2004). Conceptualizing social science paradoxes using the diversity and similarity curves model: Illustrations from the work/play and theory novelty/continuity paradoxes. Human Relations, 57(11), 1449-1477.

Pant, P. N., \& Lachman, R. (1998). Value incongruity and strategic choice. Journal of management studies, 35(2), 195-212.

Pawar, B. S., \& Eastman, K. K. (1997). The nature and implications of contextual influences on transformational leadership: A conceptual examination. Academy of Management Review, 22(1), 80-109.

Powell, T. C. (1994). Untangling the relationship between strategic planning and performance: the role of contingency factors. Canadian Journal of Administrative Sciences/Revue Canadienne des Sciences de l'Administration, 11(1), 124-138.

Ramsey, E., Ibbotson, P., Bell, J., \& Mccole, P. (2005). Internet-based business among knowledge intensive business services: some Irish regional evidence. The Service Industries Journal, 25(4), 525-545.
Reeves, T. C., Duncan, W. J., \& Ginter, P. M. (2003). Strategic configurations in health services organizations. Journal of Business Research, 56(1), 31-43.

Roberts, P. W. (1999). Product innovation, product-market competition and persistent profitability in the US pharmaceutical industry. Strategic management journal, 20(7), 655670 .

\& Greenwood, R. (1997). Integrating transaction cost and institutional theories: Toward a constrained-efficiency framework for understanding organizational design adoption. Academy of management review, 22(2), 346-373.

Schreuder, H. (1993). Timely management changes as an element of organizational strategy. Journal of Management Studies, 30(5), 723-738.

Shamsie, J., \& Mannor, M. J. (2013). Looking inside the dream team: Probing into the contributions of tacit knowledge as an organizational resource. Organization Science, 24(2), 513-529.

Small, H. (1973). Co-citation in the scientific literature: A new measure of the relationship between two documents. Journal of the American Society for Information Science, 24(4), 265-269.

Stopford, J. M., \& Baden-Fuller, C. W. (1994). Creating corporate entrepreneurship. Strategic management journal, 15(7), 521-536.

Thietart, R. A., \& Forgues, B. (1997). Action, structure and chaos. Organization Studies, 18(1), 119-143.

Valentin, E. K. (1994). Anatomy of a fatal business strategy. Journal of management studies, 31(3), 359-382.

Vogel, R., \& Güttel, W. H. (2013). The dynamic capability view in strategic management: A bibliometric review. International Journal of Management Reviews, 15(4), 426-446.

Walters, B. A., Kroll, M. J., \& Wright, P. (2007). CEO tenure, boards of directors, and acquisition performance. Journal of Business Research, 60(4), 331-338.

Yeh, Q. J., \& Fang, P. (2011). A resource-based analysis of organizational turnaround: The contingency role of organizational life 
stages. Journal of Management

Organization, 17(6), 782-796.

Yukl, G. (2009). Leading organizational learning: Reflections on theory and research. The Leadership Quarterly, 20(1), 49-53.

Zhang, C., Song, P., \& Qu, Z. (2011). Competitive action in the diffusion of internet
\& technology products in emerging markets: Implications for global marketing managers. Journal of International Marketing, 19(4), 40-60.

Zupic, I., \& Čater, T. (2015). Bibliometric methods in management and organization. Organizational Research Methods, 18(3), 429-472. 